\title{
Best practice VI Benchmarking for a better world: assessing corporate performance on the SDGs
}

\author{
Lisanne Urlings
}

\section{Description of the good practice example}

The global challenges we face, from climate disaster to gender inequality, unsustainable food systems to digital exclusion, are interconnected and affect us all. There is a perfect storm brewing with growing pressure to deliver on the United Nations' Sustainable Development Goals (SDGs) and the clock is ticking. The private sector has enormous potential to drive change. But there is currently no global accountability mechanism to understand where companies are today and where they need to get to. The World Benchmarking Alliance (WBA) has set out to develop a range of free and publicly available benchmarks to assess the progress of 2,000 keystone companies against seven systems transformations needed to achieve the SDGs (Figure VI.I).

'Business as usual' or incremental progress will not deliver the transformations needed to achieve the SDGs. It will not encourage companies to develop the innovations needed to address the challenges in the SDGs. We need business to move beyond pure financial performance and an 'avoid doing harm' mentality, to real measurable positive impact. Together with allies from government, business platforms, financial institutions, academia and civil society, WBA is developing transformative methodologies and benchmarks to measure and rank the world's most influential companies on the SDGs. WBA benchmarks produce insights, data and public rankings of these keystone companies so that investors, governments, civil society, individuals and the companies themselves better understand corporate progress and can more effectively hold companies accountable for their role in advancing the SDGs.

Companies often deal with a wide range of stakeholders with diverging expectations and priorities. Through an extensive multi-stakeholder process, benchmarks identify common ground among stakeholders and work to build consensus around these expectations. Benchmark methodologies translate these expectations into clear metrics, providing companies with 


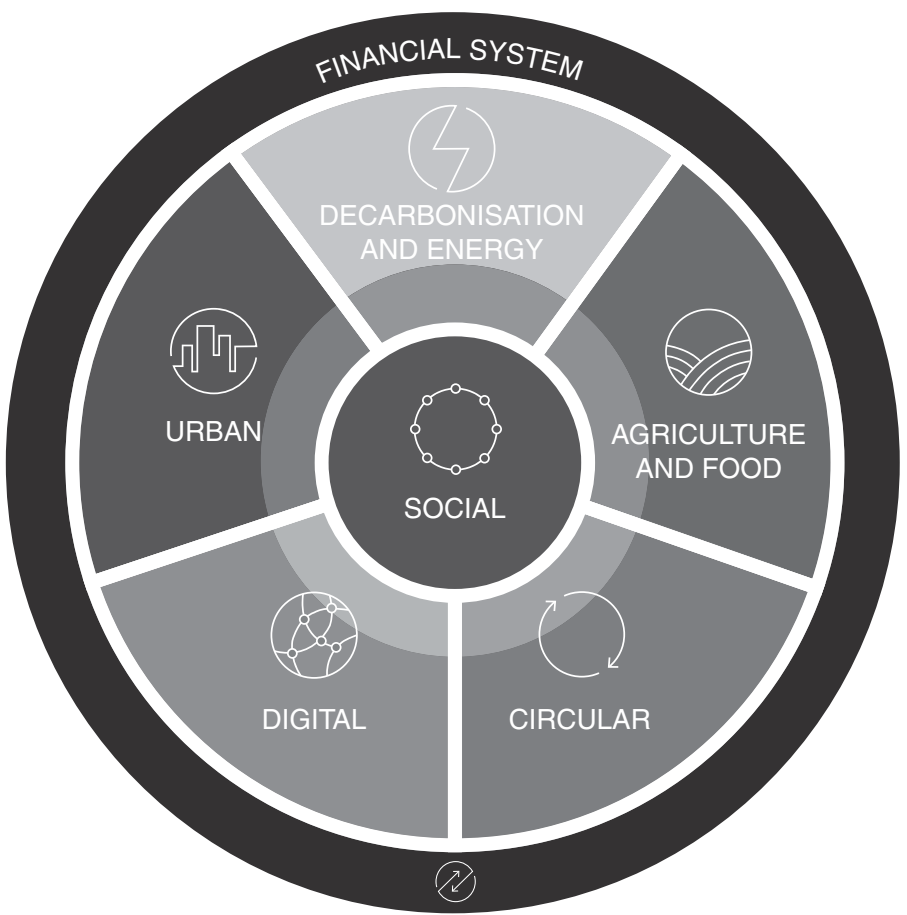

Figure VI.I World Benchmarking Alliance (WBA) seven systems transformations.

a path forward. In addition, methodologies are informed by best available science and build on existing norms and standards, frameworks and initiatives.

WBA benchmarks reveal to both companies and stakeholders where each company stands compared to its peers, where it can improve and where urgent action is needed to deliver on the SDGs in its business strategies, operations, supply chains and product and service portfolios. These benchmarks are free for everyone to use and continually improved through an open, multistakeholder dialogue. By virtue of being public and due to the way in which the data are presented, benchmarks empower all stakeholders, from consumers and investors to employees and business leaders, with key data and insights to encourage sustainable business practices across all sectors.

\section{Aims and goals}

WBA aims to drive the private sector's engagement in the SDGs through its benchmarks, envisioning a future where companies, investors, governments, 


\section{Lisanne Urlings}

civil society and individuals can quickly and easily compare businesses and motivate a 'race to the top'.

\section{Benefit for the organisation}

The benchmark results show how companies perform, both positively and negatively, in regard to the transformations and associated SDGs, show what best practice looks like, pinpoint where more action is needed and inspire innovation. 